\title{
Pretreatment predictors of malignant evolution in patients with ischemic stroke undergoing mechanical thrombectomy
}

\author{
Alessandro Davoli, ${ }^{1}$ Caterina Motta, ${ }^{1,2}$ Giacomo Koch, ${ }^{1,2}$ Marina Diomedi, ${ }^{1}$ \\ Simone Napolitano, ${ }^{1}$ Angela Giordano, ${ }^{1}$ Marta Panella, ${ }^{1}$ Daniele Morosetti, ${ }^{3}$ \\ Sebastiano Fabiano, ${ }^{3}$ Roberto Floris, ${ }^{3}$ Roberto Gandini, ${ }^{3}$ Fabrizio Sallustio ${ }^{1,2}$
}

\begin{abstract}
- Additional material is published online only. To view please visit the journal online (http://dx.doi.org/10.1136/ neurintsurg-2017-013224).

${ }^{1}$ Department of Neuroscience, Comprehensive Stroke Center, University of Tor Vergata, Rome, Italy

${ }^{2}$ Santa Lucia Foundation, Rome, Italy

${ }^{3}$ Department of Diagnostic Imaging, Interventional Radiology and Neuroradiology, University of Tor Vergata, Rome, Italy
\end{abstract}

\section{Correspondence to} Dr Alessandro Davoli, Department of Neuroscience, Comprehensive Stroke Center, University of Tor Vergata, Viale Oxford 81, 00133, Rome, Italy: aledavoli83@yahoo.com

Received 5 June 2017 Revised 10 July 2017 Accepted 14 July 2017

\section{CrossMark}

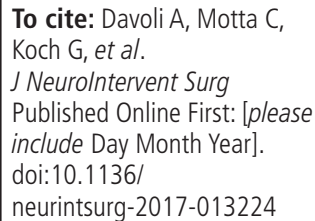

\section{ABSTRACT}

Background Few data exist on malignant middle cerebral artery infarction (MMI) among patients with acute ischemic stroke (AIS) after endovascular treatment (ET). Numerous predictors of MMI evolution have been proposed, but a comprehensive research of patients undergoing ET has never been performed. Our purpose was to find a practical model to determine robust predictors of MMI in patients undergoing ET.

Methods Patients from a prospective single-center database with AIS secondary to large intracranial vessel occlusion of the anterior circulation, treated with ET, were retrospectively analyzed. We investigated demographic, clinical, and radiological data. Multivariate regression analysis was used to identify clinical and imaging predictors of MMI.

Results 98 patients were included in the analysis, 35 of whom developed MMI (35.7\%). No differences in the rate of successful reperfusion and time from stroke onset to reperfusion were found between the MMI and nonMMI groups. The following parameters were identified as independent predictors of MMI: systolic blood pressure (SBP) on admission ( $p=0.008)$, blood glucose (BG) on admission ( $p=0.024)$, and the CTangiography (CTA) Alberta Stroke Program Early CT Score (ASPECTS) $(p=0.001)$. A scoreof $\leq 5$ in CTA ASPECTS was the best cut-off to predict MMI evolution (sensitivity $46 \%$; specificity $97 \%$; positive predictive value $78 \%$; negative predictive value $65 \%$ ).

Conclusions in our study a clinical and radiological features-based model was strongly predictive of MMI evolution in AIS. High SBP and BG on admission and, especially, a CTA ASPECTS $\leq 5$ may help to make decisions quickly, regardless of time to treatment and successful reperfusion.

\section{INTRODUCTION}

Malignant middle cerebral artery (MCA) infarction (MMI) is one of the most serious complications of ischemic stroke, leading to deterioration of consciousness over the first 24-48 hours. MMI represents between $1 \%$ and $10 \%$ of all supratentorial ischemic stroke and is characterized by a mortality rate of nearly $80 \% .^{1}$ Decompressive surgery has been shown to reduce mortality and to increase the number of patients with a favorable neurological outcome, but it is time dependent. ${ }^{2}$
Therefore, some studies have aimed to identify predictors of malignant evolution in patients with acute ischemic stroke (AIS). Imaging predictors investigated so far have mostly involved parameters such as diffusion-weighted imaging (DWI) lesions on MRI or blood-brain barrier permeability on perfusion CT. ${ }^{3-5}$ A collateral profile has already been related to DWI infarct size and clinical outcome in AIS $^{6}$ and, more recently, poor collateral circulation on multiphase CT angiography (CTA) has been shown to be an early independent predictor of MMI. ${ }^{7}$ Despite this, imaging techniques such as MRI are of limited availability in the hyperacute setting and could result in delayed MMI diagnosis. Further, the majority of studies on MMI evolution included patients with AIS receiving only intravenous recombinant tissuetype plasminogen activator (rt-PA) with a limited percentage of patients treated with endovascular treatment (ET). Therefore, we aimed to create a practical model based on demographic, clinical, and radiological findings to predict the occurrence of MMI in patients undergoing ET.

\section{METHODS}

\section{Study design and patients}

We analyzed the clinical and imaging findings of patients with AIS secondary to large intracranial vessel occlusion of the anterior circulation treated with ET from our prospective single-center database, admitted between February 2010 and December 2015 to our comprehensive stroke center.

Inclusion criteria were: (1) hospital admission within 6 hours of onset of symptoms; (2) non-contrast CT (NCCT) and CTA performed on admission; (3) proximal vessel occlusion (tandem internal carotid artery (ICA) + MCA, isolated terminal (t)-ICA or MCA main stem). Due to the study period (between 2010 and 2015) prior to publication of recent endovascular stroke trials, no exclusion criteria other than time from symptom onset were adopted until 2015. The number of excluded patients with relative causes are shown in online supplementary figure 1 .

ET was preceded by intravenous administration of rt-PA $0.9 \mathrm{mg} / \mathrm{kg}$ within 4.5 hours of stroke onset. Patients presenting beyond the time window for rt-PA administration or with major contraindications to it (ie, warfarin therapy with international normalized 
ratio 1.7 , recent major surgery, or a history of hemorrhage/hematoma) underwent stand-alone thrombectomy. No patients received intra-arterial infusion of thrombolytics.

\section{Definition of MMI}

MMI was defined as follows: (1) National Institute of Health Stroke Scale (NIHSS) score $>18$ and a level of consciousness of $\geq 1$ on item 1 a of the NIHSS, either on admission or post-admission evaluation; (2) large space-occupying MCA infarction on follow-up NCCT or MRI of at least two-thirds of the MCA territory with compression of ventricles or midline shift; and (3) no other clear cause for neurological deterioration. ${ }^{4}$

\section{Clinical assessment}

Stroke severity was assessed by a certified stroke neurologist user of NIHSS. ${ }^{8}$ Stroke etiology was classified according to the TOAST criteria. ${ }^{9}$ Demographics and vascular risk factors were reported. Clinical outcome was assessed by 90 -day modified Rankin Scale $(\mathrm{mRS})^{10}$ on outpatient clinic or telephone interview.

\section{Imaging acquisition and analysis}

The NCCT and CTA were acquired using a standardized protocol. Axial CT was performed on a multislice scanner (GE Medical Systems) using $120 \mathrm{kV}, 170 \mathrm{mAs}$ with $5 \mathrm{~mm}$ slice thickness. Continuous axial slices parallel to the orbitomeatal line were obtained from the skull base to the vertex. After this, CTA was performed using a 64-row detector. Acquisitions were obtained after a single bolus intravenous contrast injection of 90-120 mL non-ionic contrast media into an antecubital vein at 3-5 $\mathrm{mL} / \mathrm{s}$. Source images were reconstructed at $1.25 \mathrm{~mm}$ thickness in axial planes at half-thickness intervals. Before thrombectomy, in order to evaluate collaterals, an ipsilateral angiogram was obtained in the case of ICA patency whereas a contralateral angiogram was performed in the case of ICA occlusion. NCCT, CTA, and digital subtraction angiography (DSA) scans were independently reviewed by one neuroradiologist (DM) and one stroke neurologist (AD) who were blinded to the patients' symptoms but were aware of acute non-lacunar stroke. The Alberta Stroke Program Early CT Score (ASPECTS) was calculated on pretreatment NCCT and CTA. ${ }^{11} 12$

Intracranial collateral flow (ICF) was independently evaluated on CTA and DSA, adopting a scale derived from the Prolyse in Acute Cerebral Thromboembolism (PROACT) II trial for the assessment of ICF on CTA (0: no collaterals; 1 : collaterals to the periphery of ischemia; 2 : collaterals filling $50-100 \%$ of ischemic area; 3 : collaterals filling $100 \%$ of ischemic area) ${ }^{13}$ and the grading system proposed by Christoforidis et al for the assessment of ICF on DSA (based on five decreasing levels of retrograde contrast opacification of vessels within the occluded territory on delayed angiograms). ${ }^{14}$ We further dichotomized poor and good ICF (P-ICF and G-ICF), respectively, as $0-1$ or $2-3$ on CTA and 3-5 or 1-2 on DSA. The validity of this dichotomization in terms of functional outcome has already been shown. ${ }^{15}$

The hemorrhagic transformation (HT) rate was assessed according to the European Cooperative Acute Stroke Study (ECASS) criteria. $^{16}$ We evaluated reperfusion after mechanical thrombectomy according to the Thrombolysis in Cerebral Infarction (TICI) score with successful reperfusion defined as TICI $\geq 2 \mathrm{~b}$ on the final angiogram. ${ }^{17}$

\section{Statistical analysis}

Continuous variables are summarized as mean $( \pm S D)$ or median (IQR). Categorical variables are expressed as percentages (\%).
To determine differences between groups we compared the demographic, clinical, laboratory, and imaging findings using the Student's t-test or Fisher exact test, as appropriate. OR with SE and 95\% CI were provided. A p value $<0.05$ was considered statistically significant.

In order to find the best independent outcome predictors, we performed a multivariate binary logistic regression analysis with MMI as the dependent variable including, in different models, all variables with a significant association in univariate analysis. The area under the curve (AUC) of the models was calculated and the performance of the models was compared using the likelihood-ratio test, measuring the number of correct classifications to assess the improvement of model performance. Additionally, receiver operating characteristic (ROC) analysis was performed to determine the optimal CTA ASPECTS cut-off to identify patients with evolving MMI, calculating sensitivity, specificity, negative predictive value (NPV), and positive predictive value (PPV). The analysis was performed using the Stata/IC version 13 (StataCorp 2013, College Station, Texas, USA).

\section{RESULTS}

\section{Population data}

Of 133 patients with AIS, 98 were eligible for analysis; of these, 35 (35.7\%) developed MMI and 63 (64.3\%) did not (non-MMI). We found a higher systolic blood pressure (SBP) $(\mathrm{p}=0.001)$ and a higher blood glucose $(B G)$ on admission $(p=0.026)$ in the MMI group. As expected, patients with MMI presented with more severe symptoms on admission $(\mathrm{p}=0.036)$. Table 1 gives detailed clinical and demographic characteristics of both groups.

\section{Neuroimaging, treatment and outcome data}

Patients with MMI had lower NCCT ASPECTS $(\mathrm{p}<0.0001)$ and CTA ASPECTS $(\mathrm{p}<0.0001)$ and a higher rate of P-ICF on both CTA and DSA $(p=0.0001 ; p=0.0001)$. Furthermore, HT occurred more frequently in the MMI group $(\mathrm{p}<0.001)$. The use of intravenous rt-PA treatment was not significantly different between the two groups. Moreover, time from stroke onset to groin puncture and time from stroke onset to reperfusion were quite analogous. Similarly, the number of device passages and successful reperfusion according to the TICI score did not show statistically significant differences. Outcome after 90 days assessed by the mRS score showed, as expected, significant differences between the two groups $(p=0.0001)$ (table 2). Decompressive surgery was performed in $11 / 35$ (31.4\%) patients with MMI.

\section{Predictive model for MMI evolution}

The predictive model including ICF on CTA (model 2) performed significantly better than the model without them (likelihood-ratio test, $\mathrm{p}<0.001)$. Within model 2 , SBP on admission $(\mathrm{p}=0.001)$ and CTA ICF $(\mathrm{p}<0.0001)$ were identified as independent predictors of MMI, whereas BG on admission $(p=0.099)$ and NIHSS score on admission $(p=0.309)$ were not. On the other hand, if CTA ICF was not included (model 1), NIHSS score on admission $(p=0.017)$ and BG on admission $(p=0.036)$ were independent predictors of MMI, together with SBP on admission $(p=0.002)$. Adding CTA ICF to the predictive model (model 2) increased the number of correct classifications from $74.49 \%$ to 79.59\%. Analyzing a third predictive model (model 3) including CTA ASPECTS, we noticed an improvement in performance with respect to model 2 (likelihood-ratio test, $\mathrm{p}<0.001$ ). Indeed, model 3 showed how SBP on admission $(p=0.008)$ and BG on admission $(p=0.024)$ were still independent predictors of 
Table 1 Baseline clinical and demographic characteristics of patients $(n=98)$

\begin{tabular}{|c|c|c|c|}
\hline Characteristics & $\begin{array}{l}\text { MMI patients } \\
(n=35)\end{array}$ & $\begin{array}{l}\text { Non-MMI patients } \\
(\mathrm{n}=63)\end{array}$ & p Value \\
\hline Age, years, mean (SD) & $69.9( \pm 9.2)$ & $65.2( \pm 13.4)$ & 0.069 \\
\hline Sex, male & $18(51.4)$ & $30(47.6)$ & 0.833 \\
\hline NIHSS on admission, median (IQR) & $21(19-23)$ & $18(16-21)$ & 0.036 \\
\hline \multicolumn{4}{|l|}{ History } \\
\hline Arterial hypertension & $30(85.7)$ & $44(69.8)$ & 0.091 \\
\hline Diabetes mellitus & $8(22.8)$ & $11(17.4)$ & 0.596 \\
\hline Smoking & $7(20)$ & $12(19)$ & 1.0 \\
\hline Atrial fibrillation & $11(31.4)$ & $19(30.1)$ & 1.0 \\
\hline Etiology (TOAST classification) & & & 0.355 \\
\hline Cardioembolism & $17(48.5)$ & $30(47.6)$ & \\
\hline Large artery atherosclerosis & $11(34.6)$ & $19(30.1)$ & \\
\hline Other determined etiology & $0(0)$ & $5(7.9)$ & \\
\hline Undetermined etiology & $7(20)$ & $9(14.2)$ & \\
\hline SBP on admission ( $\mathrm{mm} \mathrm{Hg}$ ), median (IQR) & $160(135-184)$ & $140(130-150)$ & 0.001 \\
\hline DBP on admission (mm Hg), median (IQR) & $82(80-100)$ & $80(70-90)$ & 0.083 \\
\hline Blood glucose on admission (mg/dL), median (IQR) & $130(107-164)$ & $122(101-140)$ & 0.026 \\
\hline
\end{tabular}

Values are $\mathrm{n}(\%)$ unless indicated otherwise.

DBP, diastolic blood pressure; MMI, malignant middle cerebral artery infarction; NIHSS, National Institutes of Health Stroke Scale; SBP, systolic blood pressure.

MMI together with CTA ASPECTS $(\mathrm{p}=0.001)$, whereas NIHSS on admission $(p=0.714)$ and CTA ICF were not $(p=0.190)$. Furthermore, model 3 shows an increased number of correct classifications from $79.59 \%$ (model 2) to $82.65 \%$ (table 3). Finally, analyzing the ROC curve, we identified CTA ASPECTS $\leq 5$ as a good cut-off to predict MMI evolution in our patients (figure 1), with a low sensitivity $(46.03 \%)$ but a high specificity (97.14\%), PPV of 78\%, and NPV of $65 \%$.

\section{DISCUSSION}

Our study shows how a model including clinical findings (SBP on admission and BG on admission) and imaging findings (CTA ASPECTS) is able to predict MMI evolution in patients with anterior circulation AIS with a remarkable percentage of

Table 2 Imaging findings, treatment characteristics, and outcome data $(n=98)$

\begin{tabular}{|c|c|c|c|}
\hline & $\begin{array}{l}\text { MMI patients } \\
(n=35)\end{array}$ & $\begin{array}{l}\text { Non-MMI patients } \\
(n=63)\end{array}$ & p Value \\
\hline NNCT ASPECTS, median (IQR) & $6(4-10)$ & $9(7-10)$ & 0.0001 \\
\hline CTA ASPECTS, median (IQR) & $2(1-4)$ & $5(3-7)$ & 0.0001 \\
\hline CTA P-ICF & $24(68.5)$ & $15(23.8)$ & 0.0001 \\
\hline DSA P-ICF & $28(80)$ & $15(23.8)$ & 0.0001 \\
\hline Tandem ICA + MCA occlusion rate & $15(42.8)$ & $24(38)$ & 0.672 \\
\hline Hemorrhagic transformation & $24(68.5)$ & $20(31.7)$ & 0.001 \\
\hline Intravenous thrombolysis & $26(74.2)$ & $37(58.7)$ & 0.186 \\
\hline Time from stroke onset to groin puncture, min, mean (SD) & $234.94 \pm 62.90$ & $230.94 \pm 77.85$ & 0.794 \\
\hline Time from stroke onset to reperfusion, min, mean (SD) & $313.54 \pm 60.89$ & $312.11 \pm 80.06$ & 0.926 \\
\hline Number of device passages, mean (SD) & $2.91 \pm 1.77$ & $2.56 \pm 1.55$ & 0.300 \\
\hline Successful reperfusion $(\mathrm{TICl} \geq 2 \mathrm{~b})$ & $22(62.8)$ & $42(66.6)$ & 0.825 \\
\hline $\mathrm{mRS}$ at day 90 & & & 0.0001 \\
\hline mRS 0-2 & $0(0)$ & $31(49.2)$ & \\
\hline mRS 0-3 & $1(2.8)$ & $40(63.4)$ & \\
\hline mRS 5-6 & $27(77.7)$ & $14(22.2)$ & \\
\hline mRS 6 (dead) & $19(54.2)$ & $12(19)$ & \\
\hline
\end{tabular}

Values are $\mathrm{n}(\%)$ unless indicated otherwise.

ASPECTS, Alberta Stroke Program Early CT Score; CTA, CT angiography; DSA, Digital Subtraction Angiography; ICA+MCA, internal carotid artery+middle cerebral artery; MMI, malignant middle cerebral artery infarction; mRS, modified Rankin Scale; NNCT, non-contrast computed tomography; P-ICF, poor intracranial collateral flow; TICI, Thrombolysis In Cerebral Infarction score. 
Ischemic stroke

Table 3 Prediction of malignant MCA infarction (MMI): results of multivariate binary regression analysis with MMI as dependent variable

\begin{tabular}{|c|c|c|c|}
\hline & OR & $95 \% \mathrm{Cl}$ & $p$ Value \\
\hline \multicolumn{4}{|l|}{$\begin{array}{l}\text { Model 1: without imaging ( } n=98) \\
\text { AUC } 0.779 \text {, correct classifications: } 73 / 98(74.4 \%)\end{array}$} \\
\hline Systolic blood pressure on admission $(\mathrm{mm} \mathrm{Hg})$ & 0.970 & 0.951 to 0.989 & $0.002^{*}$ \\
\hline Blood glucose on admission (mg/dL) & 0.989 & 0.979 to 0.999 & $0.036^{*}$ \\
\hline NIHSS on admission (per point) & 0.852 & 0.748 to 0.971 & $0.017^{*}$ \\
\hline \multicolumn{4}{|l|}{$\begin{array}{l}\text { Model 2: including ICF CTA ( } n=98) \\
\text { AUC } 0.843 \text {, correct classifications: } 78 / 98(79.6 \%)\end{array}$} \\
\hline ICF CTA (dichotomized P-ICF/G-ICF) & 7.391 & 2.441 to 22.376 & $<0.0001^{*}$ \\
\hline Systolic blood pressure on admission $(\mathrm{mm} \mathrm{Hg})$ & 0.966 & 0.947 to 0.986 & $0.001 *$ \\
\hline Blood glucose on admission (mg/dL) & 0.991 & 0.981 to 1.001 & 0.099 \\
\hline NIHSS on admission (per point) & 0.926 & 0.801 to 1.072 & 0.309 \\
\hline \multicolumn{4}{|c|}{$\begin{array}{l}\text { Model 3: including ICF CTA and CTA ASPECTS ( } n=98) \\
\text { AUC } 0.892 \text {, correct classifications: } 81 / 98(82.6 \%)\end{array}$} \\
\hline CTA ASPECTS (per point) & 1.640 & 1.216 to 2.212 & $0.001^{*}$ \\
\hline ICF CTA (dichotomized P-ICF/G-ICF) & 2.372 & 0.651 to 8.647 & 0.190 \\
\hline Systolic blood pressure on admission ( $\mathrm{mm} \mathrm{Hg}$ ) & 0.971 & 0.950 to 0.992 & $0.008^{*}$ \\
\hline Blood glucose on admission (mg/dL) & 0.987 & 0.976 to 0.998 & $0.024^{*}$ \\
\hline NIHSS on admission (per point) & 0.970 & 0.824 to 1.141 & 0.714 \\
\hline
\end{tabular}

${ }^{*} \mathrm{p}<0.05$.

ASPECTS, Alberta Stroke Programme Early CT Score.; CTA, CT angiography; G-ICF, good intracranial collateral flow; ICF, intracranial collateral flow; NIHSS, National Institutes of Health Stroke Scale; P-ICF, poor intracranial collateral flow.

correct classifications (82.65\%). SBP on admission has already been associated with an increased infarct volume and lower rate of 3-month favorable outcome in patients with large vessel occlusion treated with ET. ${ }^{18}$ BG on admission, even in non-diabetic patients, together with failure in glycemia drop in the first 48 hours, are both significant predictors of 90-day poor outcome and death ${ }^{19}$; moreover, high $\mathrm{BG}$ on admission is predisposed to $\mathrm{HT} .{ }^{20}$ Our study confirmed these findings, together with a high rate of HT (68.5\%). Regarding imaging data, we found CTA ASPECTS as the unique independent predictor of MMI evolution and the most powerful predictor of the entire model, significantly increasing the percentage of correct classifications. In recent studies, CTA ASPECTS was found to better predict final infarct and outcome compared with NCCT ASPECTS. ${ }^{12}$ For this

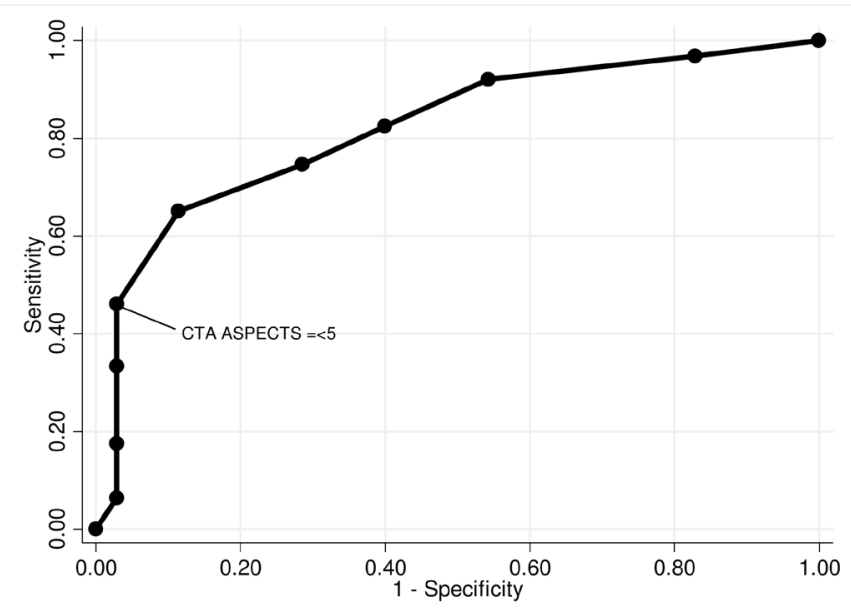

Figure 1 Receiver operating characteristic curve for the prediction of malignant middle cerebral artery infarction by CTA ASPECTS. Corresponding sensitivity/specificity of $46 \% / 97 \%$ for CTA ASPECTS $\leq 5$. reason we decided to include only CTA ASPECTS in our model, even if in univariate analysis NCCT ASPECTS was also significantly lower in the MMI patient group. Furthermore, the cut-off of CTA ASPECTS $\leq 5$ better predicted MMI evolution, with a remarkably high sensitivity $(97.14 \%)$. With regard to this, a recent study identified a score of $\leq 5$ on CTA ASPECTS as the best cut-off to predict futile recanalization. ${ }^{21}$ Interestingly, our study groups did not differ in terms of successful reperfusion, corroborating the concept of futile ET in patients with low CTA ASPECTS; similarly, the MMI and non-MMI groups showed no differences in stroke onset to groin puncture time and stroke onset to reperfusion time, confirming that malignant evolution of stroke seems to be independent of ET successful reperfusion and time to reperfusion. In our model ICF status failed to remain an independent predictor of $\mathrm{MMI}$ after the addition of CTA ASPECTS, even though patients with MMI showed a P-ICF profile more frequently than non-MMI patients. It is well known that P-ICF correlates with poor outcome. ${ }^{1522}$ For this reason and in order to create a model useful for making early decisions in clinical practice, we included in the multivariate analysis only ICF status on CTA. Severity of neurological deficit on admission assessed by the NIHSS has already been suggested as a predictor of MMI, ${ }^{23}$ and even in our study the MMI group was characterized by a significantly higher NIHSS. However, in our logistic regression model, NIHSS failed to be an independent predictive factor. Interestingly, our groups had a similar rate of tandem occlusion, potentially predictive of MMI, and of patients treated with intravenous thrombolysis. Therefore, intravenous thrombolysis does not seem to affect the malignant evolution of stroke or HT.

Our study has several limitations. The long period of data collection with improved expertise and the use of different protocols and devices over the years could have created heterogeneity in patient treatment, leading to potential bias. The rate of MMI in our study was higher than in previous reports; nevertheless, 
our analysis focused on large vessel anterior circulation stroke as demonstrated by admission NIHSS, NNCT ASPECTS and age, which make any comparison with recent endovascular stroke trials and previous malignant stroke studies unreliable. A further limitation is represented by the lack of other neuroimaging data such as pretreatment DWI volume or perfusion CT mismatch. However these technologies, unlike CTA, are not available in all stroke centers, especially in the emergency setting.

\section{CONCLUSIONS}

Our study shows that a practical model including admission SBP, BG, and CTA ASPECTS (with a cut-off $\leq 5$ ) may quickly and accurately predict MMI in a subgroup of patients with AIS treated with ET, regardless of time to treatment and successful reperfusion.

Contributors AD: study concept/design; analysis/interpretation of data; acquisition of data; study supervision/coordination; obtaining funding; drafting/revising the manuscript for content. CM: analysis/interpretation of data; drafting/revising the manuscript for content; statistical analysis. GK: study concept/design; analysis/ interpretation of data; obtaining funding; study supervision/coordination. MD: analysis/interpretation of data; drafting/revising the manuscript for content. SN: analysis/interpretation of data; acquisition of data. AG: analysis/interpretation of data; acquisition of data. MP: analysis/interpretation of data; acquisition of data. DM: analysis/interpretation of data; acquisition of data. SF: analysis/interpretation of data; acquisition of data. RF: analysis/interpretation of data; drafting/revising the manuscript for content; study supervision/coordination. RG: analysis/interpretation of data; drafting/revising the manuscript for content, acquisition of data. FS: study concept/design; analysis/interpretation of data; drafting/revising the manuscript for content; obtaining funding; study supervision/coordination; acquisition of data.

Funding This work was supported by Italian Ministry of Health grant number RF2013-02358679.

\section{Competing interests None declared.}

Patient consent Obtained.

Ethics approval Local ethics committee.

Provenance and peer review Not commissioned; externally peer reviewed.

(c) Article author(s) (or their employer(s) unless otherwise stated in the text of the article) 2017. All rights reserved. No commercial use is permitted unless otherwise expressly granted.

\section{REFERENCES}

1 Hacke W, Schwab S, Horn M, et al. 'Malignant' middle cerebral artery territory infarction: clinical course and prognostic signs. Arch Neurol 1996;53:309-15.

2 Vahedi K, Hofmeijer J, Juettler E, et al. Early decompressive surgery in malignant infarction of the middle cerebral artery: a pooled analysis of three randomised controlled trials. Lancet Neurol 2007;6:215-22.

3 Bektas H, Wu TC, Kasam M, et al. Increased blood-brain barrier permeability on perfusion CT might predict malignant middle cerebral artery infarction. Stroke 2010;41:2539-44.

4 Thomalla G, Hartmann F, Juettler E, et al. Clinical Trial Net of the German Competence Network Stroke. Prediction of malignant middle cerebral artery infarction by magnetic resonance imaging within 6 hours of symptom onset: a prospective multicenter observational study. Ann Neurol 2010;68:435-45.

5 Minnerup J, Wersching $\mathrm{H}$, Ringelstein EB, et al. Prediction of malignant middle cerebral artery infarction using computed tomography-based intracranial volume reserve measurements. Stroke 2011;42:3403-9.

6 Souza LC, Yoo AJ, Chaudhry ZA, et al. Malignant CTA collateral profile is highly specific for large admission DWI infarct core and poor outcome in acute stroke. AJNR Am J Neuroradiol 2012;33:1331-6.

7 Flores A, Rubiera M, Ribó M, et al. Poor collateral circulation assessed by multiphase computed tomographic angiography predicts malignant middle cerebral artery evolution after reperfusion therapies. Stroke 2015;46:3149-53.

8 Brott T, Adams HP, Olinger CP, et al. Measurements of acute cerebral infarction: a clinical examination scale. Stroke 1989;20:864-70.

9 Adams HP, Bendixen BH, Kappelle LJ, et al. Classification of subtype of acute ischemic stroke. Definitions for use in a multicenter clinical trial. TOAST. Trial of Org 10172 in Acute Stroke Treatment. Stroke 1993;24:35-41.

10 van Swieten JC, Koudstaal PJ, Visser MC, et al. Interobserver agreement for the assessment of handicap in stroke patients. Stroke 1988;19:604-7.

11 Barber PA, Demchuk AM, Zhang J, et al. Validity and reliability of a quantitative computed tomography score in predicting outcome of hyperacute stroke before thrombolytic therapy. ASPECTS Study Group. Alberta Stroke Programme Early CT Score. Lancet 2000;355:1670-4.

12 Sallustio F, Motta C, Pizzuto S, et al. CT Angiography ASPECTS Predicts Outcome Much Better Than Noncontrast CT in Patients with Stroke Treated Endovascularly. AJNR Am J Neuroradiol 2017.

13 Tan JC, Dillon WP, Liu S, et al. Systematic comparison of perfusion-CT and CTangiography in acute stroke patients. Ann Neurol 2007;61:533-43.

14 Christoforidis GA, Mohammad Y, Kehagias D, et al. Angiographic assessment of pial collaterals as a prognostic indicator following intra-arterial thrombolysis for acute ischemic stroke. Am J Neuroradiol 2005;26:1789-97.

15 Sallustio F, Motta C, Pizzuto S, et al. CT angiography-based collateral flow and time to reperfusion are strong predictors of outcome in endovascular treatment of patients with stroke. J Neurointerv Surg 2016 (Published Online First: 23 September 2016. doi: 10.1136/neurintsurg-2016-012628).

16 Hacke W, Kaste M, Fieschi C, et al. Randomised double-blind placebo-controlled trial of thrombolytic therapy with intravenous alteplase in acute ischaemic stroke (ECASS II). Second European-Australasian Acute Stroke Study Investigators. Lancet 1998;352:1245-51.

17 Higashida RT, Furlan AJ, Roberts H, et al. Technology Assessment Committee of the American Society of Interventional and Therapeutic Neuroradiology; Technology Assessment Committee of the Society of Interventional Radiology. Trial design and reporting standards for intra-arterial cerebral thrombolysis for acute ischemic stroke. Stroke 2003;34:e109-37.

18 Goyal N, Tsivgoulis G, Iftikhar S, et al. Admission systolic blood pressure and outcomes in large vessel occlusion strokes treated with endovascular treatment. J Neurointerv Surg 2017;9:451-4.

19 Natarajan SK, Dandona P, Karmon Y, et al. Prediction of adverse outcomes by blood glucose level after endovascular therapy for acute ischemic stroke. J Neurosurg 2011;114:1785-99.

20 Paciaroni M, Agnelli G, Caso V, et al. Acute hyperglycemia and early hemorrhagic transformation in ischemic stroke. Cerebrovasc Dis 2009;28:119-23.

21 Kawiorski MM, Martínez-Sánchez P, García-Pastor A, et al. Alberta Stroke Program Early CT Score applied to CT angiography source images is a strong predictor of futile recanalization in acute ischemic stroke. Neuroradiology 2016;58:487-93.

22 Liebeskind DS, Tomsick TA, Foster LD, et al. Collaterals at angiography and outcomes in the Interventional Management of Stroke (IMS) III trial. Stroke 2014;45:759-64.

23 Krieger DW, Demchuk AM, Kasner SE, et al. Early clinical and radiological predictors of fatal brain swelling in ischemic stroke. Stroke 1999;30:287-92. 


\section{JIS Pretreatment predictors of malignant evolution in patients with ischemic stroke undergoing mechanical thrombectomy}

Alessandro Davoli, Caterina Motta, Giacomo Koch, Marina Diomedi, Simone Napolitano, Angela Giordano, Marta Panella, Daniele Morosetti, Sebastiano Fabiano, Roberto Floris, Roberto Gandini and Fabrizio Sallustio

J Neurolntervent Surg published online August 10, 2017

Updated information and services can be found at:

http://jnis.bmj.com/content/early/2017/08/05/neurintsurg-2017-01322

4

These include:

References This article cites 21 articles, 12 of which you can access for free at: http://jnis.bmj.com/content/early/2017/08/05/neurintsurg-2017-01322 4\#BIBL

Email alerting Receive free email alerts when new articles cite this article. Sign up in the service box at the top right corner of the online article.

Topic Articles on similar topics can be found in the following collections Collections Ischemic stroke (397)

\section{Notes}

To request permissions go to:

http://group.bmj.com/group/rights-licensing/permissions

To order reprints go to:

http://journals.bmj.com/cgi/reprintform

To subscribe to BMJ go to:

http://group.bmj.com/subscribe/ 